\title{
Corela
}

Cognition, représentation, langage

HS-3 | 2006

Les verbes d'apparence

\section{Juger d'après les apparences : le cas du français}

\section{Alain Delplanque}

\section{OpenEdition}

\section{Journals}

Édition électronique

URL : http://journals.openedition.org/corela/1284

DOI : $10.4000 /$ corela. 1284

ISSN : 1638-573X

\section{Éditeur}

Cercle linguistique du Centre et de l'Ouest - CerLICO

\section{Référence électronique}

Alain Delplanque, « Juger d'après les apparences : le cas du français », Corela [En ligne], HS-3 | 2006, mis en ligne le 13 mars 2006, consulté le 19 avril 2019. URL : http://journals.openedition.org/ corela/1284 ; DOI : 10.4000/corela.1284

Ce document a été généré automatiquement le 19 avril 2019

\section{(c) (i) (2)(2)}

Corela - cognition, représentation, langage est mis à disposition selon les termes de la licence Creative Commons Attribution - Pas d'Utilisation Commerciale - Partage dans les Mêmes Conditions 4.0 International. 


\title{
Juger d'après les apparences : le cas du français
}

\author{
Alain Delplanque
}

Je remercie, outre mes collègues de Tours, ma relectrice Sarah de Vogüé dont les remarques ont largement contribué à clarifier et approfondir ma réflexion.

1 Loin de prétendre couvrir la totalité des expressions de l'apparence en français, je me concentrerai ici sur «sembler, ressembler, avoir l'air, faire». Ces verbes ont ceci de commun qu'ils traduisent un jugement de l'énonciateur sur l'état d'un sujet donné, avec une certaine modulation qui les oppose à l'attribution franche :

\begin{tabular}{|l|l|}
\hline 1 Pierre semble triste & Pierre est triste \\
\hline 2 Marie ressemble à une sorcière & Marie est une sorcière \\
\hline 3 Ton écharpe fait jolie & Ton écharpe est jolie \\
\hline 4 Jacques a l'air de dormir & Jacques dort \\
\hline
\end{tabular}

2 En même temps, ces verbes d'apparence se distinguent des verbes de constat ou de jugement, dans la mesure où ceux-ci sont explicitement construits à partir d'un sujet énonciateur visant un être du monde réel :

5 Je vois que Pierre est triste

6 Je trouve (que) ton écharpe (est) jolie

3 Alors que, dans l'expression de l'apparence, l'énonciateur évacue sa place de " constructeur », quitte à réapparaître, dans certains cas et dans certaines conditions, comme « récepteur » du procès :

7 Pierre m'a l'air triste

8 Ton écharpe me semble jolie 
Cette mise en retrait du constructeur du jugement instaure un "décalage » entre l'énonciation et la représentation du réel, phénomène qui pourrait être le dénominateur commun à tous les verbes d'apparence. Cela dit, la diversité même de ces expressions nous interpelle et nous invite à dégager, par delà leurs points communs, la spécificité de chacune. Celle-ci ne peut sérieusement être dégagée qu'à partir des propriétés distributionnelles de chaque expression. Encore faut-il interpréter correctement de telles propriétés. Je vais tenter de le faire dans la perspective des opérations prédicatives et énonciatives (A. Culioli 1990). Du point de vue prédicatif, ces expressions, en tant que verbales, expriment une lexis, dotée d'un schéma spécifique, qui ne réfère à rien en ellemême, mais qui marque une opération portant sur une seconde lexis, ici appelée « complément » et représentant le procès principal qui suit.

$\langle a r b\rangle$ verbe d'apparence $\langle x \vee y\rangle$

procès complément

Le premier terme de ces lexis $(\mathrm{a}, \mathrm{x})$ sera appelé « source » et le second terme $(\mathrm{b}, \mathrm{y})$ « but », la dénotation $\mp$ agentive ou $\mp$ locative de ces mots dépendant du type de verbe utilisé ${ }^{~}$. Du point de vue énonciatif, la spécificité de chaque schéma devra rendre compte de la manière dont les paramètres énonciatifs sont diversement activés ou pondérés. Je souligne que tous ces verbes d'apparence (figurant dans la première lexis) expriment des " états ", au sens où ils sont incompatibles avec les marques de processus.

* Pierre est en train de (me) sembler triste

* Marie est en train de ressembler à une sorcière

* Ton écharpe est en train de faire jolie

* Jacques est en train d'avoir l'air de dormir

Mais ces verbes n'instaurent pas la même relation inter-sujets, et ne peuvent pas tous être suivis d'un complément au datif: "Pierre me semble bon». En outre, ces verbes diffèrent quant à leur compatibilité avec tel ou tel type de complément (figurant dans la seconde lexis) : adjectival, nominal, verbal sous forme infinitive ou verbal sous forme complétive : « Il semble que Pierre dorme ».

$7 \quad$ La forme et le sens de l'énoncé global résultent de l'articulation de ces deux lexis. Ceci est essentiel pour comprendre comment on peut, à travers un verbe d'apparence intrinsèquement statif, appréhender un état ou un processus, un complément continu ou discontinu.

\section{1. « sembler » et « avoir l'air »}

Ces deux verbes d'apparence ont en commun la possibilité d'être suivis d'un complément non-verbal (adjectif ou nom) ou d'un complément verbal :

\begin{tabular}{|l|l|}
\hline 9 Pierre semble triste & \\
Pierre a l'air triste & \\
Ce travail semble une montagne & 10 Pierre semble aimer le chocolat \\
Ce nuage a l'air d'une montagne & \\
Le chat a l'air d'aimer le chocolat & \\
\hline
\end{tabular}


Ils ont également en commun leur compatibilité avec la marque du «datif» de l'observateur, interprété ici comme un énonciateur. Cet actant particulier sera noté $\mathrm{S}$ dans le schéma de lexis du verbe d'apparence $<\mathrm{X} \mathrm{r} \mathrm{S}>$

\begin{tabular}{|l|l|}
\hline 11 Pierre me semble triste & 12Pierre m'a l'air triste \\
Ce travail me semble une montagne & Ce type m'a l'air d'un casse-pieds \\
Le chat me semble dormir & Le chat m'a l'air de dormir \\
\hline
\end{tabular}

10 En comparant ces deux verbes avec «faire, ressembler ", on constate que la compatibilité d'un verbe d'apparence avec un complément verbal coïncide avec la compatibilité de ce verbe avec le datif de l'observateur:

\begin{tabular}{|l|l|}
\hline $13 *$ Pierre me ressemble à Paul & $14 *$ Pierre me fait triste \\
\hline$*$ Pierre ressemble à dormir & $*$ Pierre fait dormir \\
\hline
\end{tabular}

11 Comment expliquer cette corrélation? Un verbe est, par définition, une notion conjugable car il exprime un ensemble de propriétés (simultanées ou successives) devant être validées dans l'espace-temps de l'énonciateur. Alors que le nom et l'adjectif réfèrent à un ensemble de propriétés atemporelles qui ne peuvent être validées que du point de vue qualitatif. C'est pourquoi la présence de l'énonciateur est constitutive de la lexis de "sembler, avoir l'air »: elle est nécessaire en tant que repère ordonnant les paramètres énonciatifs So, To qui structurent la seconde lexis :

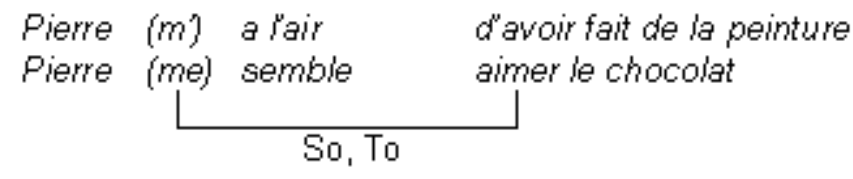

12 La lexis étant une structure sous-jacente représentant les propriétés du verbe, il va de soi que cette corrélation est constante, même quand ce repère $S$ est sous-entendu en surface. On peut donc dire que si ces deux expressions « sembler, avoir l'air » sont bel et bien des états, elles possèdent néanmoins dans leur format notionnel le repère énonciatif qui les rend compatibles avec un processus.

\subsection{Le sujet impersonnel}

13 La première différence entre ces deux verbes est que "sembler» peut avoir un sujet impersonnel, contrairement à « avoir l'air »

\begin{tabular}{|l|l|l}
\hline 15 & Il semble que le chat soit endormi & $*$ \\
\hline
\end{tabular}

14 Qu'est-ce qu'un sujet impersonnel ? D'une manière générale, l'impersonnel est l'indice d'un sujet « vide » qui renvoie à un sujet réel non ancré dans la situation d'énonciation. On recourt notamment à la construction impersonnelle lorsque le sujet est indéfini : 


\begin{tabular}{|l|l|}
\hline 16 Un policier est venu & 17 Le policier est venu \\
$\rightarrow$ Il est venu un policier & $*$ Il est venu le policier \\
$*$ du vin est à la cave & Le vin est à la cave \\
$\rightarrow$ Il y a du vin à la cave & $*$ Il y a le vin à la cave \\
$*$ de la neige tombe & La neige tombe \\
$\rightarrow$ Il tombe de la neige & $*$ Il tombe la neige \\
\hline
\end{tabular}

Dans ces exemples, le sujet impersonnel renvoie au sujet réel qui est syntaxiquement déplacé. Plus généralement, ce sujet vide marque un décalage entre un prédicat préconstruit et son ancrage dans l'espace énonciatif :

\begin{tabular}{|l|l|}
\hline 18 Travailler est utile & 19 Que Pierre vienne est possible \\
\hline$\rightarrow$ Il est utile de travailler & $\rightarrow$ Il est possible que Pierre vienne ${ }^{3}$ \\
\hline
\end{tabular}

16 Dans le cas de "sembler ", la source de la lexis est vide, car ce verbe exprime un décalage entre le plan de l'énonciation et le plan du préconstruit, que j'appellerai ici celui de la " co-énonciation ». Ce concept ne doit pas être confondu avec l'interlocution. Il s'agit de l'altérité subjective qui est fondatrice de toute énonciation, mais qui se manifeste de manière plus ou moins éclatante selon le degré de modalisation de l'énoncé.

\section{Sito Sit'o}

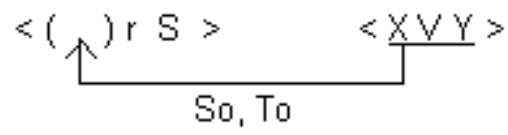

\section{II me sembie que Pierre n'est pas sorti \\ II ne semble pas que Pierre soit sorti}

17 Les deux exemples ci-dessus montrent que, dans cette énonciation dédoublée, chacun des deux prédicats peut porter l'assertion Toutefois la validité du préconstruit est soumise à son degré d'intégration dans l'énonciation. C'est ainsi que le verbe de la proposition complément apparait au subjonctif, sauf en présence du datif de l'observateur :

20 Il semble que Pierre soit sorti

* Il me semble que Pierre soit sorti

$\rightarrow$ Il $\underline{\text { me }}$ semble que Pierre est sorti

Dans cette construction, la proposition complément est donc clairement subordonnée à la première (présence du connecteur « que »). Mais ce n'est là qu'une disposition possible en surface, car au niveau sous-jacent, la co-énonciation permet d'envisager les deux prédicats comme virtuellement interdépendants au sein de l'énoncé, ce qui explique la possibilité d'avoir une construction détachée (incise) :

21 Pierre est sorti, ce me semble

ou Pierre, semble-t-il, est sorti. 
19 Simplement, l'incise a ceci de particulier qu'elle ne peut porter la négation (* Pierre est sorti, ce ne me semble pas), car alors le procès principal n'est pas préconstruit en Sit'o mais construit au premier plan de l'énonciation Sito.

\subsection{Le sujet personnel}

Il en va tout autrement avec « avoir l'air ». Cette fois, dans la première lexis, la source de la relation prédicative est nécessairement un objet du monde, à partir duquel l'énonciateur infère le procès complément. Ainsi, le sujet de "avoir l'air " peut être considéré comme la source d'une relation dont l'énonciateur est le but. De ce fait, la notion d'«air » qui, avec l'auxiliaire «avoir », traduit un état, peut avec l'auxiliaire approprié exprimer un processus, volontaire ou involontaire : « prendre un air, prendre des airs »- ce qui est inconcevable avec « sembler ».

21 De plus, à la différence de « sembler », le décalage ici est instauré, non entre un prédicat préconstruit et son ancrage dans l'espace énonciatif, mais entre les propriétés perceptibles d'un objet (l'air qu'il $\mathrm{a}=\mathrm{X}$ ) et ses propriétés réelles, inconnues de l'énonciateur (ce qui est représenté par la parenthèse vide ci-dessous) : on ne dit pas que Pierre aime, mais qu'il en a l'air.

Sito

\section{Sit2}

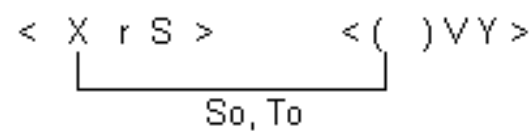

\section{Pierre m'a lair d"aimer le chocolat \\ Pierre n'a pas yair d'aimer le chocolat}

22 Ici le procès «aimer" est en quelque sorte en attente d'un sujet. Il n'est donc pas conjugable, sauf à dire : Il a l'air de qqun qui aime le chocolat. Certes, ce procès peut être envisagé de manière positive ou négative (Pierre a l'air de ne pas aimer le chocolat). Mais il ne s'agit pas là d'une assertion, mais d'une simple référence à l'Intérieur ou à l'Extérieur du domaine notionnel qui dépend de la représentation qu'on se fait du réel (Sit2) : ne pas aimer = manger peu, faire la grimace, etc. Contrairement à «sembler », l'assertion est donc effectuée dans une énonciation non-dédoublée. D'ailleurs, cette fois-ci, le prédicat complément n'a aucune autonomie syntaxique par rapport au prédicat d'apparence, auquel il est totalement subordonné (incise impossible) :

$22 *$ Pierre, il a l'air, aime le chocolat

* Pierre aime le chocolat, a-t-il l'air.

Par ailleurs, le « datif » de l'observateur n'a pas les mêmes latitudes avec « avoir l'air » et avec « sembler ». Avec « sembler », étant donné le dédoublement énonciatif (Sito - Sit'o), cet observateur peut coïncider avec l'origine du propos, ou être voisin de lui, ou être séparé de lui (respectivement 1ère, 2ème, ou 3ème personne grammaticale). Alors qu'avec " avoir l'air », l'observateur au datif coïncide nécessairement avec l'énonciateur-origine de l'énoncé (1ère personne grammaticale), car l'énonciation n'est pas dédoublée : 
$S=$ So Marie me semble dormir

$S \underline{\underline{E}}$ So Marie te semble dormir

$S \omega$ So Marie lui semblait dormir
Marie m'a l'air de dormir

? Marie t'a l'air de dormir?

* Marie lui avait l'air de dormir est d'emblée ancré dans l'espace-temps de l'énonciation défini par le verbe d'apparence dont il dépend. Ainsi, lorsque le complément Y est un substantif, la double activation des paramètres So, To confère à cette notion des contours physiquement définis, ce qui appelle normalement un complément concret et discret :

23 Ton tas de sable a l'air d'une montagne (sens propre)

? Ton tas de sable semble une montagne

24 Ce travail lui semblait une montagne (sens figuré)

? Ce travail avait l'air d'une montagne

Alors que le schéma correspondant à " sembler » suggère que le procès complément < X V $\mathrm{Y}>$ n'est pas directement ancré dans l'espace-temps de l'énonciation. De fait, lorsque le complément $\mathrm{Y}$ est un substantif, ce terme ne peut réfèrer à un objet aux contours concrètement définis dans l'espace. Il s'agit pratiquement toujours soit d'une abstraction («ça semble une utopie, une évidence, un scandale, un obstacle, un succès, une bonne idée ... »), soit d'un nom pris au sens figuré (« ça semble une montagne, il semble le roi de la soirée, Sylvie semble la perle de toutes les candidates... ») :

25 Leur mariage semble une erreur (abstrait)

? Leur mariage a l'air d'une erreur

26 L'automobile semble la clé de leur économie (sens figuré)

? L'automobile a l'air de la clé de leur économie

Le bout de fer ainsi limé a l'air d'une clé (sens propre)

Ce phénomène peut s'expliquer en termes de co-énonciation : les compléments nominaux de « sembler » sont des occurrences fictives, apparentées à la comparaison hypothétique : ce travail, c'est aussi difficile que si c'était une montagne; ce mariage c'est comme si c'était une erreur, etc.

\subsection{La construction du sujet syntaxique}

Les schémas ci-dessus sont incomplets dans la mesure où le sujet syntaxique ne correspond pas forcément à la source de la lexis sous-jacente. Il suffit pour s'en convaincre de se souvenir des phénomènes de voix (sujet source / sujet but). Cette distinction sujet/ source permet peut-être d'expliquer l'instabilité de l'accord grammatical lorsque "avoir l'air» est suivi d'un adjectif. Comme je l'ai suggéré, l'apparence implique un certain fractionnement du sujet: on a d'une part, le sujet apparent (son « air » X) et d'autre part, le sujet « réel » qu'on peut seulement imaginer, ce que j'ai noté par la parenthèse vide. L'accord de l'adjectif se fera avec l'un ou avec l'autre, selon que la propriété est envisagée comme passagère ou inhérente :

27 Cette fille a l'air boudeuse, angoissée

Cette fille a un air boudeur, angoissé

Le complément ne pourra être verbalisé avec «être » que dans le premier cas : «Elle a l'air d'être boudeuse, angoissée ». 
délicat est le double comportement du verbe « sembler » qui peut avoir soit un sujet impersonnel soit un sujet personnel. Dans ce dernier cas, deux questions se posent, à savoir (i) si la distinction «sembler / avoir l'air » est alors vraiment neutralisée ; (ii) sinon, quelle est la différence entre les deux constructions personnelles. Je suppose ici que "sembler» est fondamentalement un verbe impersonnel, ce qui le différencie de " avoir l'air », même dans les constructions à sujet personnel.

\begin{tabular}{|c|c|c|c|}
\hline () & $\begin{array}{l}\langle\text { () r } S\rangle \\
\text { semble }\end{array}$ & que & $\begin{array}{l}\langle X V Y\rangle \\
\text { la porte soit verrouilée }\end{array}$ \\
\hline $\begin{array}{l}X \\
\text { La porte }\end{array}$ & $\begin{array}{l}\langle\text { () r } S\rangle \\
\text { semble }\end{array}$ & & $\begin{array}{l}\langle X \vee Y\rangle \\
\text { (être) verrouillée }\end{array}$ \\
\hline porte & $\begin{array}{l}\langle\mathrm{X} \text { r So }\rangle \\
\text { a l'air }\end{array}$ & & $\begin{array}{l}\langle() \vee Y\rangle \\
\text { (d'être) verrouilée }\end{array}$ \\
\hline
\end{tabular}

Certes, la nuance n'est pas toujours éclatante; mais elle apparaît plus nettement dès l'instant où le sujet est inaccessible au regard de l'énonciateur. Par exemple, si l'énonciateur est un inspecteur de police et Pierre l'occupant d'un appartement faisant l'objet d'une perquisition

28 La porte semble verrouillée de l'intérieur

La porte a l'air verrouillée de l'intérieur

29 Pierre semble avoir verrouillé de l'intérieur

? ? ? Pierre a l'air d'avoir verrouillé de l'intérieur

31 Si l'on compare le jeu des parenthèses vides dans les schémas de lexis respectifs de nos deux verbes, on voit qu'avec « sembler », le sujet X n'est pas ancré en Sito, mais en Sit'o ; on a donc un décalage entre une hypothèse imaginée en Sit'o et sa recevabilité par l'énonciateur en Sito, indépendamment de la présence de X. Alors qu'avec " avoir l'air ", le sujet est ancré en So, To ; on a donc un prédicat qui est directement inféré d'après les propriétés perceptibles d'un objet $\mathrm{X}$ nécessairement présent.

\section{Le cas du verbe « faire »}

Dans la plupart de ses acceptions, le verbe «faire » exprime une action, c'est-à-dire un processus qui se déroule dans le temps et qui est déclenché par une source (l'agent qui entre en activité) : «Pierre fait son lit, Pierre fait le clown, Pierre me fait rire ». On peut même dire que «faire » est le processus par excellence, puisqu'il est l'archisémème de l'ensemble des verbes d'action: «Qu'est-ce que tu fais dans la vie?»C'est cette propriété qui autorise son usage anaphorique, là où il est parfois appelé verbe vide : "Je travaille plus que tu ne le fais ». L'intégration de « faire » dans notre champ d'étude pourrait donc surprendre le lecteur. Elle est pourtant justifiée, en raison des acceptions comme 30-31 qui, dans d'autres langues, sont bel et bien traduites par un verbe d'apparence :

30 Pierre fait triste dans son nouveau costume (he looks sad)

31 Pierre fait clown dans son nouveau costume (he looks like a clown)

\subsection{Compactage du domaine notionnel}

Il faut reconnaître que « faire » se distingue des deux verbes précédents à bien des égards. D'abord, il ne peut être suivi d'un verbe : 
32 Pierre semble dormir

Pierre a l'air de dormir

33 * Pierre fait dormir, dormant, dormi

$\rightarrow$ il me donne l'impression de dormir...

Ensuite, contrairement à "sembler, avoir l'air ", il ne peut être suivi du datif de l'observateur. Sinon, il n'exprime plus l'apparence mais la sensation :

34 * Pierre me fait triste dans son nouveau costume

35 Pierre me fait mal ; il me fait plaisir ;

il me fait froid dans le dos; votre réponse me fait chaud au cœur.

D'après ce que nous avons vu avec «sembler, avoir l'air ", on comprend que ces deux incompatibilités vont de pair : l'absence de $\mathrm{S}$ dans la première lexis prive la seconde lexis du repérage spatio-temporel nécessaire aux procès verbaux. On peut donc dire que, dans l'expression de l'apparence, « faire » désactive à la fois le paramètre inter-sujets So (datif impossible) et le paramètre aspectuel To (complément verbal impossible). Corollairement, alors que les deux autres verbes admettent l'opposition aspectuelle accompli / inaccompli, «faire» ne peut exprimer l'apparence qu'à l'inaccompli (passé composé incongru) : 4

\begin{tabular}{|l|l|}
\hline 36 Pierre me semblait triste & Pierre m'a semblé triste \\
\hline 37 Pierre avait l'air triste & Pierre a eu l'air triste \\
\hline 38 Pierre faisait triste & * Pierre a fait triste \\
\hline
\end{tabular}

Finalement, «faire » n'a en commun avec les deux précédents que la possibilité d'être suivi d'un attribut adjectival. On remarquera notamment que les deux autres verbes peuvent être suivis d'un nom avec article: "il a l'air d'un clown, cela semble une montagne ». Alors que si l'attribut de « faire » est un substantif, il ne peut apparaitre que sans article, donc avec une valeur adjectivale, car si le nom est doté d'un article, «faire » n'est plus un verbe d'apparence mais un verbe d'action : «Il fait le clown ».

Il y a deux manières de référer à une qualité, selon la prépondérance de tel ou tel paramètre énonciatif, que je noterai par un jeu de parenthèses. Avec «sembler \& avoir l'air ", le domaine notionnel de l'énoncé est «discret", car les paramètres spatiotemporels prédominent sur le qualitatif - ce que je noterai (Sito) So, To ${ }^{5}$. Dans ce cas, l'attribution d'une qualité est effectuée en référence à une occurrence-type : «Pierre a l'air d'un clown». Tandis qu'avec «faire », le domaine de l'énoncé est « compact», avec une prépondérance du point de vue qualitatif sur le spatio-temporel : Sito (So, To). Dans ce cas, la prédication réfère à ce qu'A. Culioli appelle l'attracteur, c'est-à-dire la qualité pure: "Pierre fait clown». Et je souligne que cette référence à l'intérieur strict du domaine notionnel peut se faire indépendamment de toute occurrence, et même parfois en dépit de la réalité concrète. Effectivement, quand je dis «Pierre fait triste », je ne dis pas que Pierre « est » triste ; il n'y a pas identification d'une propriété inhérente au sujet, mais invention d'une propriété à propos de ce sujet, compte tenu de son environnement ou de son comportement. Disons que "faire" traduit un jugement d'après les apparences. Cette pseudo-attribution sera ici représentée par un verbe vide dans la seconde lexis $\langle\mathrm{X}() \mathrm{Y}\rangle$. 


\subsection{Le schéma de lexis de «faire»} occulté pour que le verbe cesse d'exprimer un processus :

42 C'est alors que «faire » peut intervenir : « le gigot fait $3 \mathrm{~kg}$ ». Seulement, ce verbe étant vide, l'idée de mesure devient alors implicite : schéma de lexis $\langle\mathrm{X}(\mathrm{)}) \mathrm{Y}\rangle$. Souvent, la référence est récupérable à partir des propriétés de $\mathrm{X}$ ou $\mathrm{Y}:$ : le madrier fait $4 \mathrm{~m}$ » évoque a priori la longueur du madrier. Mais ce n'est pas toujours aussi évident: «le trou fait $1 \mathrm{~m}$ » est ambigu car la dimension peut référer à la longueur, à la largeur ou à la profondeur. Quoi qu'il en soit, dans l'esprit des locuteurs, «faire» est associé à une 
relation prédicative implicite spécifiant les conditions de l'évaluation, tout en occultant l'évaluateur, qui sera ici représenté par une parenthèse vide dans la première lexis :

\begin{tabular}{|l|l|}
\hline$<() \mathrm{r} \mathrm{X}>$ & $<\mathrm{X}(\mathrm{)} \mathrm{Y}$ \\
\hline (on pèse $\mathrm{X})$ & le gigot fait $3 \mathrm{~kg}$ \\
\hline (on prend la longueur de $\mathrm{X})$ & le madrier fait $4 \mathrm{~m}$ \\
\hline
\end{tabular}

43 D'une manière générale, on peut dire que quand « faire » est un verbe d'état, il exprime un procès dépouillé de son contenu spatio-temporel au profit de l'évaluation quantitative ou qualitative. ${ }^{6}$ Ainsi, lorsque "faire» exprime une apparence, il est en relation de paraphrase avec un verbe d'évaluation (trouver, penser, considérer, se représenter comme) ; acception explicitement mentionnée dans les dictionnaires: «On le fait plus riche qu'il ne l'est ». La source de ces verbes est un énonciateur portant un jugement sur le terme but du point de vue strictement qualitatif.

\begin{tabular}{|l|l|}
\hline$<\underline{\mathrm{S}} \mathrm{V} \mathrm{X}>\langle\mathrm{X}() \mathrm{Y}\rangle$ & $<() \mathrm{r} \mathrm{X}><\mathrm{X}() \mathrm{Y}\rangle$ \\
\hline 42 je trouve Pierre débraillé & Pierre fait débraillé \\
\hline 43 je trouve ce gilet très snob & ce gilet fait très snob \\
\hline
\end{tabular}

Cette configuration rend compte de la duplicité du terme $\mathrm{X}$ qui a été évoquée plus haut. D'un côté, le schéma $<\mathrm{X}(\mathrm{)}) \mathrm{Y}>$ rend l'idée que l'objet $\mathrm{X}$ est la source d'un certain effet. Il ne s'agit pas tant d'une « action » que cet objet exercerait sur l'observateur que de l'effet produit par l'interaction entre cet objet et son environnement $<$ ( ) r X > : Avec le lit, la pièce fait moins vide ". D'une certaine manière, cet effet est comparable à celui d'une addition :

44 Deux cafés et un croissant, ça fait trois euros.

45 Dix euros, ça fait cher pour deux cafés et un croissant.

De l'autre côté, le schéma < ( ) r X > rend l'idée que X est le but d'une relation dont l'évaluateur est la source, même si celui-ci est implicite. Le gigot ne pèse $3 \mathrm{~kg}$ que parce qu'on l'évalue comme tel (avec balance ou à vue de nez). De même, Pierre n'est débraillé que dans la mesure où on le juge débraillé. Je souligne que l'absence de marque de l'énonciateur $\mathrm{S}$ ne signifie pas une absence de subjectivité, mais simplement une absence de repérage spatio-temporel (voir: "avoir l'air, sembler »). On peut même dire que «faire» traduit le plus souvent des verdicts à forte subjectivité, soit laudatifs, soit péjoratifs :

46 ça fait chic, sympa, bien, super

47 ça fait ringard, beauf, plouc, pédé.

\subsection{Apparence et jugement}

Le verbe «faire " s'écarte ainsi radicalement de "sembler ", dans la mesure où la lexis qu'il introduit n'est pas une prédication pré-construite, mais une relation prédicative en 
quelque sorte en attente de validation $\langle\mathrm{X}(\mathrm{)}) \mathrm{Y}\rangle$. Ce qui explique pourquoi le verbe " faire » ne peut en aucun cas être suivi d'une proposition complétive (* Il fait que Pierre est triste). L'attribution effective de la propriété $\mathrm{Y}$ au sujet $\mathrm{X}$ est assumée dans une énonciation non-dissociée (Sito $=$ Sit'o). En termes pragmatiques, cette absence de coénonciation rend compte de l'intuition que le jugement ici rendu est sans appel et en dehors de tout débat. Ce jugement arbitraire est motivé davantage par l'imaginaire de l'énonciateur que par les données physiques de l'objet. Le décalage est ici instauré entre l'état réel des choses (en S2, T2) et la représentation que l'énonciateur se donne de la notion qualifiante $Y$ (en Sit2)

$$
\text { S2, T2 }
$$

\section{Sit2}

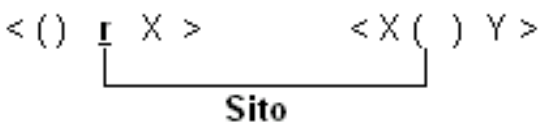

\section{Sans le lit, la pièce elle fait vide}


l'interprétation figurée, tandis qu'avec l'article, le complément de "avoir l'air » prend obligatoirement son sens propre et concret :

$52 \mathrm{Tu}$ fais clown avec ce costard $=\mathrm{Tu}$ as l'air clown avec ce costard

$\neq$ Tu as l'air d'un clown avec ce costard

$53 \mathrm{Ce}$ costume fait très anglais $=$ ce costume $\mathrm{a}$ l'air très anglais

Ce costume fait très Anglais $\rightarrow^{*}$ ce costume a l'air d'un Anglais

L'exemple 53 montre qu'avec «faire ", l'adjectif peut qualifier le costume ou, de manière métonymique, son porteur: le paramètre qualitatif est activé indépendamment de l'ancrage spatio-temporel. Lorsque la notion n'a pas de sens figuré ou de stéréotype particulier, le nom sans article prend valeur générique. Alors que « avoir l'air » suivi d'un $\mathrm{N}$ avec article exprime une simple comparaison, l'attribution en «faire » sans article, elle, relève de la caricature :

\begin{tabular}{|l|l|}
\hline 54 Sa maison fait très ranch du Far-west & Sa maison a l'air d'un ranch du Far-west \\
\hline $55 \mathrm{Tu}$ fais cuisinier avec cette toque & Tu as l'air d'un cuisinier avec cette toque \\
\hline 56 Sa coiffure fait palmier sur la tête & Sa coiffure a l'air d'un palmier sur la tête \\
\hline
\end{tabular}

53 Mais cette fois, « avoir l'air » souffre très difficilement la suppression de l'article, car il y aurait dans ce cas un conflit entre la double activation So,To de « avoir l'air » et la double occultation (So, To) résultant de l'adjectivation du nom :

$$
\begin{aligned}
& 57 \text { * Sa maison a l'air ranch du Far-west } \\
& 58 \text { * Sa coiffure a l'air palmier sur la tête } \\
& 59 \text { ? Tu as l'air cuisinier avec cette toque }
\end{aligned}
$$

Ce compactage explique sans doute pourquoi ce genre de tournure est meilleur avec une qualification supplémentaire : "Il a l'air bon cuisinier ». Mais justement, dans ce cas, la relation spatio-temporelle ne peut être réactivée: * Il a l'air bon cuisinier avec cette toque. Cette malléabilité ne vient pas de « avoir l'air » en tant que tel, mais de la diversité des compléments que ce verbe peut prendre.

\section{Le cas du verbe « ressembler »}

Ce verbe se distingue radicalement de tous les verbes précédents. Aussi peut-on se demander, ici encore, si ce verbe mérite de figurer dans une étude de l'apparence. Je l'ai pourtant intégré dans la présente étude pour diverses raisons. D'abord, parce que la ressemblance se distingue de l'identité par un décalage qui rappelle celui de l'apparence : " ça ressemble à de l'or, mais ce n'est que du plaqué ». Par ailleurs, certains exemples étudiés en russe, en anglais et en allemand se traduisent spécifiquement par "ressembler" ("she looks like Madonna»). De plus, en français, on peut difficilement faire fi de la parenté étymologique qui existe entre "ressembler" et "sembler". Et nous aurons à rendre compte de certaines complémentarités intéressantes entre ce verbe et "avoir l'air » et « faire ». 


\subsection{Densité de la « ressemblance »}

61 Mais la ressemblance n'est pas une comparaison. Celle-ci active clairement le paramètre qualitatif et peut notamment être suivie d'un adjectif : " Il était comme fou, je me sens comme fatigué » - contrairement à la ressemblance. On peut donc dire que la 
ressemblance crée, en elle-même, un domaine notionnel typiquement dense, où ne prédomine ni le paramètre inter-personnel, ni le paramètre spatio-temporel, ni le qualitatif.?

$\begin{array}{llll}\text { Faire } & \text { Sito } & (\text { So, To }) & \text { compact } \\ \text { Awoir l'air } & \text { (Sito) } & \text { So, To } & \text { discret } \\ \text { Ressembler } & \text { (Sito) } & \text { (So, To) } & \text { dense }\end{array}$

\section{2. statut du complément}

62 Cette densité affecte l'ensemble de l'énoncé. On constate justement que "ressembler » peut être suivi d'un complément dense (massif), alors que « avoir l'air » ne peut être suivi que d'un nom discret:

\begin{tabular}{|l|l|}
\hline 67 ça ressemble à du sucre & $*$ ça a l'air du sucre \\
\hline 68 Ça ressemble à un morceau de sucre & $\rightarrow$ ça a l'air d'un morceau de sucre \\
\hline
\end{tabular}

63 Lorsqu'au contraire, le complément $Y$ est un nom discret, les compatibilités dépendent du repérage spatio-temporel. L'article indéfini marque une référence au "type » et dans ce cas, les deux verbes sont quasi synonymes. Mais si le complément $Y$ est défini, l'article ne renvoie plus au type, mais à une occurrence délimitée en co-énonciation (mention préalable). Dans ce cas, « avoir l'air » est exclu :

\begin{tabular}{|l|l|}
\hline 69 Jean ressemble à un grand'père & Jean a l'air d'un grand'père \\
\hline (occurrence quelconque) & (type) \\
\hline 70 Jean ressemble à son grand'père & * Jean a l'air de son grand'père \\
\hline (occurrence spécifique et définie) & (type) \\
\hline
\end{tabular}

Corollairement, seul «ressembler» est autorisé lorsque le complément est un nom propre ou une personne au sens grammatical du terme, mots qui renvoient forcément à une occurrence et non à un type :

\begin{tabular}{|l|l|}
\hline 71 Pierre ressemble à Marie & * Pierre a l'air de $\underline{\text { Marie }}$ \\
\hline 72 Pierre me ressemble ; & * Pierre a l'air de moi ; \\
c'est une boutique qui vous ressemble & $*$ cette boutique a l'air de vous \\
\hline
\end{tabular}

De plus, la comparaison figurée (par métaphore ou par métonymie) n'est possible qu'à condition de faire abstraction d'une partie des propriétés de l'objet. Cela est impossible avec «avoir l'air », car la référence au type présuppose une congruence sémantique que ces figures ne respectent pas. Alors qu'avec « ressembler », la mise en relation de $\mathrm{X}$ et $\mathrm{Y}$ 
est justement effectuée sur la base d'une propriété implicite, ce qui donne une marge de manœuvre beaucoup plus grande :

73 Un auteur ressemble à son œuvre

l'histoire de la Corse ressemble à sa géographie

74 * un auteur a l'air de son œuvre ;

* l'histoire de la Corse a l'air de sa géographie.

Par ailleurs, lorsque la comparaison est effectuée entre deux termes d'une même classe, la qualité commune allant de soi, c'est le rapport entre les occurrences qui prédomine: ressemblance dans ce cas. Mais avec « avoir l'air », il y aurait contradiction entre l'idée de centrage autour d'un type et la dispersion des occurrences de la même espèce :

$75 \mathrm{Ce}$ vin ressemble à tous les autres ;

sa carrière ressemble à la mienne

76 ? Ce vin a l'air de tous les autres ;

? sa carrière a l'air de la mienne

On peut alors se demander ce qui se passe lorsque le complément est tautologique, contexte favorisant la neutralisation de nos deux verbes :

77 Chez Georges, le menu ressemble à un menu; ton château ressemble à un château

78 Chez Georges, le menu a l'air d'un menu ; ton château a l'air d'un château

Avec "ressembler", on a un effet de banalisation du sujet parmi les concurrents partageant la même propriété : c'est un menu (un château) comme les autres, sans plus ${ }^{8}$. Tandis qu'avec "avoir l'air", on a un effet de valorisation, comme si la conformité au type était non garantie. Selon le contexte discursif, la glose sera: c'est un menu (un château) jusqu'à preuve du contraire, ou: c'est bel et bien un menu (c'est un vrai château). En même temps, ces deux verbes construisent deux scénarios énonciatifs différents. Avec « avoir l'air », les interlocuteurs se trouvent (ou s'imaginent) en présence de l'objet. Tandis que "ressembler » exprime l'apparence indépendamment de cette présence. Ceci est d'autant plus intéressant lorsqu'il s'agit d'un objet générique dans un discours didactique, comme ceci :

79 le daman ressemble à un lapin, exception faite de son anatomie interne.

80 le daman a l'air d'un lapin, mais il a une denture de cheval.

Le premier énoncé ci-dessus est un exemple réel tiré d'un article de l'encyclopédie Encarta, où il s'agit de taxinomie zoologique. Le second énoncé est fabriqué : le ton serait moins docte, comme si l'on cherchait à détromper l'interlocuteur.

Enfin, lorsque le complément $Y$ réfère à un ensemble vide, les effets sont conformes à la pondération des paramètres énonciatifs. Quand on dit (81a), ce qui est évoqué est le complémentaire spatio-temporel: on sous-entend «mais c'est quelque chose ou c'est beaucoup ». Alors que si on dit (81b), on n'évoque pas l'altérité, mais la nullité : la qualité est anéantie en même temps que la classe d'occurrences est vidée.

81a ça n'a l'air de rien

$81 \mathrm{~b}$ ça ne ressemble à rien

\subsection{Le préfixe « re - »}

71 Le verbe « ressembler » se distingue de son étymon "sembler » qui, comme nous l'avons vu, ne peut être suivi d'un nom que si celui-ci est abstrait ou pris au sens figuré (le type), alors que « ressembler» ne permet d'envisager qu'un nom concret au sens propre (une occurrence) 


\begin{tabular}{|l|l|}
\hline 82 Pierre ressemble à un clown & ? ? Pierre semble un clown \\
\hline 83 Pierre ressemble à un monstre & Pierre semble un monstre \\
\hline 84 ? ? ? Cette fête ressemble à un prétexte & cette fête semble un prétexte \\
\hline
\end{tabular}
poète n'aurait sans doute pas été jusqu'à dire: «? Le canon ressemblait à la voix de Bonaparte ", mais plutôt « Le canon tonnait comme si c'était la voix de Bonaparte, il me semblait que le canon tonnait comme la voix de Bonaparte...» En un mot, avec « ressembler», le complément $Y$ ne peut être qu'une occurrence dotée d'une qualité implicite. sémantique diachronique et synchronique. Quelle est la valeur de ce préfixe re- qui bouleverse à ce point les propriétés énonciatives du radical? Pour comprendre, nous disposons notamment de deux articles qui ont eu le mérite de transcender les acceptions temporelles classiques (« répétition », comme dans « relire » et « inversion » comme dans «revenir »). Ne pouvant ici analyser ces deux articles en détail faute de place, je me contenterai de l'essentiel.

74 Selon P. Jalenques (2001), la valeur temporelle de répétition n'est qu'un cas particulier, non généralisable. Il formule la valeur de re- comme marquant une « modification » $\mathrm{P} 1 \rightarrow$ P2 dans l'espace-temps de l'énonciation. Pour JJ. Franckel (1989), re-P marque la construction d'une occurrence de $\mathrm{P}$ dont la délimitation extrinsèque s'opère «à partir d'un premier ancrage de P dans le temps ». Dans les deux cas, on reconnaît que reimplique deux prédications opposées appréhendées en deux temps. L'idée de modification ne suffit pas, car re- exprime beaucoup plus qu'une simple transformation. $\mathrm{Au}$ contraire re- exprime bien une «dissociation» entre ce qui est fait et ce qui néanmoins reste à faire. En guise de synthèse, voici ce que je propose pour caractériser le sens de ce préfixe :

$\mathrm{RE}+\mathrm{V}$ marque une relation contradictoire entre la source et le but du procès $\mathrm{V}$.

Cette contradiction s'entend d'abord du point de vue inter-sujets, dans la mesure où la source $\mathrm{X}$ est dans une position problématique vis-à-vis de son but $\mathrm{Y}$ : ceci apparaît notamment lorsque RE a un sens inversif : «Pierre revient à Paris » = Pierre a quitté Paris + Pierre vient à Paris. Cette contradiction est aussi à prendre au sens qualitatif dans la mesure où $\mathrm{RE}+\mathrm{V}$ marque l'annulation d'un état validé préalablement à la réalisation du procès $\mathrm{V}$. Autrement dit, RE marque la conversion d'un Intérieur en Extérieur, à partir duquel un nouvel Intérieur est visé. Ceci est applicable à l'exemple précédent, mais également en l'absence d'effet d'inversif : « Marie raccourcit sa robe » = quelle que soit la dimension de départ (position IE), la robe est d'abord jugée comme non-courte (position E) avant d'envisager le processus qui la rendra courte (position I). Dans tous les cas, le but visé est un nouveau point de référence que se donne l'énonciateur. Enfin, si le verbe exprime un processus, la contradiction est repérée dans la classe des instants : hors du temps énonciatif, on abolit l'état1 (construction d'une position E); dans le temps énonciatif, on vise l'état2 (construction d'une position I). Le terme du processus peut être 
différent du point de départ (valeur itérative : « relire le texte ») ou identique au point de départ (valeur inversive : « revenir à Paris ») :

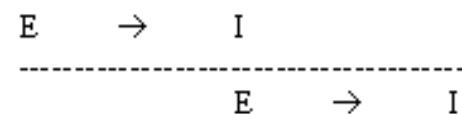

re-lire t.1

$\mathrm{t} 2$

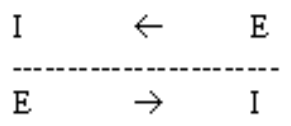

re-venir

Les cas de RE préfixé à un verbe compact sont rares. J'en connais deux, à savoir « reconnaître » et « ressembler» qui justement nous préoccupe ici. Je vais tenter de montrer comment ces deux verbes sont à la fois comparables et opposés. Il va de soi qu'aucun de ces deux verbes n'exprime ni la répétition, ni l'inversion; mais dans les deux cas, on présuppose une relation contradictoire « $\mathrm{A} \hookleftarrow \mathrm{B}$ », telle que A et B sont à la fois identiques et différents par rapport à certaines propriétés. Ce qui oppose ces deux verbes, c'est la manière dont l'énonciateur traite cette relation contradictoire.

Le sujet de «re + connaître" est un énonciateur S. Et ce verbe implique que l'identification d'un objet $\mathrm{X}$ est a priori compromise par le fait que cet objet partage avec son concurrent $X^{\prime}$ un grand nombre de propriétés. Mais l'énonciateur $S$ parvient à séparer les deux termes grâce à une propriété différentielle Y pouvant être explicitées dans le contexte :

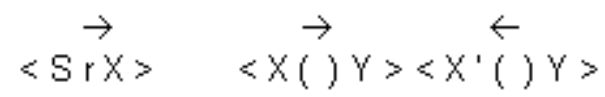

85 on reconnaît le lièvre $\mathrm{X}$ à ses pattes $\mathrm{Y}$

(par rapport au lapin X')

Avec «sembler", rappelons-le, l'énonciateur est clairement but-récepteur de l'apparence, dans un schéma de lexis $\langle$ ( ) r S >, ce qui permet le datif et un complément verbal. Tandis qu'avec le dérivé «re + sembler », ce repère n'est pas utile car les paramètres So, To sont inactivés (ni datif, ni complément verbal). Je dirais même que ce repérage est impossible car l'énonciateur ne peut ou ne veut s'exprimer ni comme source, ni comme but de la représentation. Il est à la fois récepteur (il est frappé par la ressemblance) et constructeur (il trouve une ressemblance). Dans ces conditions, l'énonciateur ne peut que s'effacer.

Par ailleurs, chacun des deux termes X et $Y$ étant support de la même propriété, le choix du sujet syntaxique est ici indifférent. Il est à cet égard remarquable que " ressembler » est le seul de nos verbes qui permette la réciprocité de $\mathrm{X}$ et $\mathrm{Y}$ :

86 Pierre ressemble à Marie

Marie ressemble à Pierre

Pierre et Marie se ressemblent

Nous nous retrouvons ainsi avec une lexis sans actants dont la seule valeur est d'exprimer l'ambiguité propre à la ressemblance, l'énonciateur ne pouvant ou ne voulant pas stabiliser la relation entre $\mathrm{X}$ et $\mathrm{Y}$ :

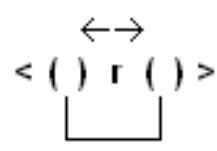

Sito

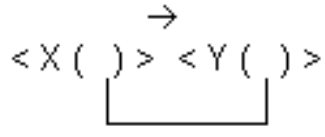

Sit2, S2, T2 
87 le laiton ressemble à de l'or

(c'est jaune et brillant, mais c'est moins cher)

\subsection{La préposition}

81 En fin de compte, c'est la préposition «à » qui marque l'orientation du domaine. D'une manière générale, une préposition exprime un repérage dans l'espace-temps de l'énonciateur. De ce fait, avec « faire », la relation prédicative étant compacte, le domaine notionnel ne possède aucune frontière permettant d'envisager la relation $\mathrm{X}-\mathrm{Y}$ comme une occurrence; pas de localisation spatio-temporelle, pas de préposition. Les verbes noncompacts, au contraire, exigent une préposition « à » ou « de $»^{9}$ :

$88 \mathrm{Tu}$ fais clown avec cette cravate

$89 \mathrm{Tu}$ as l'air d'un clown avec cette cravate

$90 \mathrm{Tu}$ ressembles à un clown avec cette cravate préposition « de " marque une relation exclusive, et "à » une relation non-exclusive : "L'amie de Pierre / une amie à Pierre; un gâteau de riz / un pain au chocolat». Lorsqu'on travaille sur la classe des instants, « de » marque un processus accompli et « à » un processus inaccompli : «Pierre commence à écrire, continue à écrire / il finit d'écrire, il vient d'écrire ». Enfin, lorsqu'on travaille sur une relation assertable, « de » marque une validation effective et « à » une validation virtuelle : « un tasse de café / une tasse à café ; décider de faire / hésiter à faire ». On peut généraliser en disant que " de » marque une relation, dans un domaine discret, entre un intérieur et un extérieur intrinsèquement séparés par une frontière. C'est ainsi que "Pierre est de Paris » situe le sujet dans l'extérieur d'un domaine centré par rapport à l'énonciateur. Alors que «à » marque une relation, dans un domaine dense, entre deux points voisins situés soit dans la frontière (« il va à Paris ») soit dans l'intérieur strict du domaine notionnel (« il est à Paris »).

D’une manière plus générale, étant donné l'absence de délimitation intrinsèque, dans un domaine dense, l'intérieur coïncide avec la frontière ${ }^{10}$. Résumons-nous par un schéma :

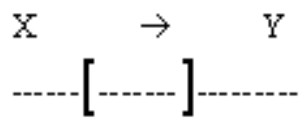

de

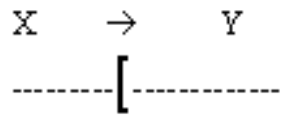

à

Avec « avoir l'air de », la relation X - Y construit un domaine discret; ce domaine est discontinu car il est muni d'une frontière bien délimitée permettant d'envisager une occurrence centrée sur l'intérieur qualitatif. La délimitation est conforme au double repérage To (complément verbal possible) et So (l'énonciateur évoque un type extérieur par rapport à X). Tandis qu'avec « ressembler à ", la prédication est dense, au sens où les deux termes $\mathrm{X}$ et $\mathrm{Y}$ sont mis en relation dans le même intérieur notionnel (la propriété implicite). La non-délimitation correspond à l'absence de temporalité, mais aussi à l'absence de type. 


\section{Apparence, apparition, parution}

86 permet de distinguer des phénomènes étymologiquement liés, à savoir : l'apparence et l'apparition-parution.

\subsection{Le verbe « apparaître »}

87 Ce verbe a deux sens et deux types de constructions syntaxiques. Du côté de l'apparition, il exprime un processus signifiant «devenir visible» et compatible avec toutes les marques aspectuelles :

91 Une certaine subjectivité ne serait-elle pas en train d'apparaître?

92 Le trafic de drogue est en voie d'expansion. De nouvelles menaces sont en train d'apparaître sur le marché.

93 Et c'est alors que le génie apparut à Aladin.

On voit que dans ce sens, ce verbe peut être utilisé soit de manière absolue, soit avec un complément de lieu, soit avec un complément récepteur. Du côté de l'apparence, le même verbe exprime un état dont le complément récepteur peut être exprimé ou sousentendu :

94 Ce qui nous est intérieur (nous) apparaît aujourd'hui obscur, solitaire et informe.

95 Son échec devant Claude Farrère apparut à beaucoup comme un scandale.

Dans ce sens-là, "apparaitre " a pratiquement la même distribution que le verbe " sembler » étudié ci-dessus. Avec un sujet personnel, il peut être suivi d'un adjectif, d'un nom ou d'un verbe ; avec un sujet impersonnel, il est suivi d'une complétive :

96 Le contrôle policier ne nous apparaît pas présenter les garanties nécessaires.

97 Très vite il m'apparut que Hugo occupait une place de choix

La différence entre ces deux verbes est d'ordre temporel, puisque "apparaitre » est employé dans des contextes qui indiquent que le jugement sur les apparences intervient dans un deuxième temps, au terme d'une étude ou d'une période de réflexion, ou du moins dans des conditions spatio-temporelles autres que celles qu'on a pu ou qu'on pourrait imaginer :

98 Au terme de l'étude de faisabilité d'un télescope de 5 mètres, il apparut très vite qu'une combinaison de SCHWARZSCHILD nécessiterait une coupole trop importante et trop onéreuse.

99 La connaissance d'une deuxième langue étrangère est un atout incontestable qui apparaît même de plus en plus comme une nécessité.

100 Beaucoup se demandent quel est le rapport entre les deux premières catéchèses et celle-ci. Personnellement, le lien m'apparaît évident.

$101 \mathrm{Il}$ avait semblé que le pont était d'une solidité à toute épreuve. Mais au cours de l'enquête, il apparut très vite que les normes de sécurité n'avaient pas été respectées

91 Ce décalage temporel est un invariant du verbe " apparaitre ». Dans le sens d'apparition, il marque le passage de l'invisible au visible dans l'espace concret d'un énonciateur. Dans le sens d'apparence, il marque le passage dans le champ de la connaissance d'un énonciateur. Ce décalage temporel est absent des usages de « sembler ». 


\subsection{Le verbe " paraître »}

92 Ce verbe, tout comme " apparaître », peut traduire soit une apparition (102: processus), soit une apparence (103: état) avec des compléments comparables à ceux de « sembler » :

102 Le soleil parut à l'horizon

103 Pierre paraît intelligent

Le monde qu'il évoque paraît une utopie

La sentinelle paraît dormir à poings fermés

Ce verbe est étymologiquement lié à « apparaître », mais il n'a pas exactement les mêmes propriétés que ce dernier. En tant que processus, le verbe "paraître " est une notion dense, exprimant une activité non délimitée dans le temps. Alors que " apparaître » est un verbe discret, exprimant un changement de lieu. En l'occurrence, ces deux verbes ne forment pas le passé composé de la même manière :

104 Alors, le soleil est apparu

$105 *$ Alors, le soleil est paru $\rightarrow$ le soleil a paru

94 Ces propriétés aspectuelles vont de pair avec la transitivité. Dans le schéma de lexis d'un verbe dense, c'est le terme source qui est prépondérant; le terme but étant facultatif et même parfois exclu. Alors qu'avec un verbe discret, le terme prépondérant est le but de la lexis ${ }^{11}$. Ceci rend compte d'abord de l'incompatibilité de "paraître " avec le datif de l'observateur lorsqu'il traduit un processus :

\begin{tabular}{ll} 
Paraître & Apparaître \\
$\langle\mathrm{Xr}(\mathrm{X})$ & $\langle(\mathrm{X}) \mathrm{r} \mathrm{S}\rangle$ \\
\hline 106 Le soleil (*nous) parut à l'horizon & Le soleil nous apparut à l'horizon \\
\hline
\end{tabular}

Cela dit, le verbe «paraître " permet un jeu tout à fait particulier sur l'activation du paramètre inter-sujets. En effet, lorsque "paraître " est pris au sens d'apparence, le schéma de lexis est alors re-formaté en $\langle\mathrm{X} \mathrm{r} \mathrm{S}\rangle$, avec un terme but comme celui de « avoir l'air » - ce qui n'est guère surprenant puisque ce glissement de sens s'obtient par « compactage » du verbe d'apparition :

107 Pierre me paraît intelligent

Le monde qu'il évoque me paraît une utopie

En échange, en raison même de la prépondérance de la source dans le schéma de lexis de " paraître ", la construction impersonnelle constitue une déformation plus importante et qui n'est pas sans conséquences. Si l'on introduit un but explicite (datif de l'observateur), le sens d'apparence est possible, mais cette tournure < ( ) r S > est rare, ressentie comme soutenue, voire archaïque. En français actuel, on lui préfère «sembler», de schéma identique :

$108 \mathrm{Ami}$, il me paraît que ta dialectique est aussi fausse que ton esprit. (Sade)

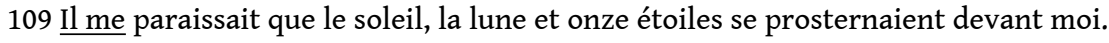

(Bible)

97 Mais en l'absence de but, la lexis se vide de tout actant < ( ) r ( ) > et n'exprime plus ni apparition, ni apparence, mais indique le caractère non-testimonial de l'assertion. Cette expression impersonnelle prend alors le sens d'un verbe de parole mais dont le sujet 
serait indéfini : «il parait que = on dit que ». Elle devient en même temps une modalité d'assertion, proche de certains usages du conditionnel en français :

110 Il (*me) paraît que les épreuves ont été annulées

= on dit que les épreuves ont été annulées

= De source bien informée, les épreuves auraient été annulées

Ceci est un effet de la prégnance particulière de la source dans le schéma de lexis d'origine du verbe "paraitre ». Le vidage actanciel n'est pas sans rappeler les flèches à double sens qui caractérisaient «faire, ressembler »; mais cette fois, il s'agit de marquer un décalage entre deux origines du propos, (So - S1) l'énonciateur et le locuteur, ce dernier se posant à la fois comme récepteur et émetteur :

$$
<\text { (S1) } \overrightarrow{\mathbf{r}}(\mathrm{So})>
$$

Ce phénomène est d'autant plus frappant qu'inversement, le verbe «dire », utilisé au conditionnel et avec sujet indéfini, est équivalent à nos verbes d'apparence :

111 On dirait que la sentinelle dort à poings fermés.

= il semble que la sentinelle dorme à poings fermés

= la sentinelle a l'air de dormir à poings fermés.

\section{Conclusion}

En définitive, les verbes d'apparition, en tant que verbes de processus, ne concernent l'apparence que de manière dérivée. Les verbes d'apparence proprement dits sont compacts et instaurent un décalage entre deux états. Plus précisément :

- «sembler » marque un décalage entre une proposition XVY préconstruite et sa validation dans la situation dénonciation.

- " ressembler » marque un décalage entre les propriétés partagées par deux sujets X et $\mathrm{Y}$ et les propriétés qui les distinguent.

- "avoir l'air » marque un décalage entre les propriétés perceptibles d'un sujet X et l'idée qu'on se fait de la propriété Y.

- «faire » marque un décalage entre la situation objective de $\mathrm{X}$ et le jugement $\mathrm{Y}$ qu'on formule à son propos.

Ce décalage étant marqué par des verbes de valence différente, j'ai tenté de représenter chaque type d'apparence par un schéma de lexis spécifique. De sorte que toutes les propriétés distributionnelles de chaque construction découlent de l'articulation de la première lexis (apparence) avec la lexis qui la suit (complément). C'est ainsi que chaque schèma établit une corrélation entre le rôle donné à l'énonciateur et la pondération des paramètres énonciatifs au sein de l'énoncé global.

En ce qui concerne le premier point, on a deux cas de figure. Soit l'énonciateur se pose explicitement comme le constructeur de la prédication; c'est le cas du jugement: « je trouve que Pierre ... ». Soit l'énonciateur se pose comme récepteur de la prédication ; c'est ce type de décalage qui définit l'apparence : «Pierre me semble ..., il mª l'air de ... » . Les verbes «faire » et « ressembler» constituent des cas hybrides exprimant un jugement d'après les apparences. En ce qui concerne le second point, on a dégagé trois types de comportement: discret ("avoir l'air» et "sembler»), compact («faire») et dense ( ressembler »), selon que le complément peut être verbal, strictement adjectival ou strictement nominal. 


\begin{tabular}{|c|c|c|c|}
\hline sembler & avoir l'air de & faire & ressembler à \\
\hline$\langle 0) r S\rangle\langle X \vee Y\rangle$ & $\langle X r S\rangle\langle 0) \vee Y\rangle$ & $\langle 0 r X\rangle\langle X() Y\rangle$ & $\left.\left\langle(\vec{r}()\rangle\left\langle X_{0}\right)\right\rangle\left\langle Y_{0}\right\rangle\right\rangle$ \\
\hline $\begin{array}{c}\text { Discret } \\
\text { (Sito) So, To }\end{array}$ & $\begin{array}{c}\text { Discret } \\
\text { (Sito) So, To }\end{array}$ & $\begin{array}{c}\text { Compact } \\
\text { Sito }\left(\mathrm{So}_{\mathrm{T}} \mathrm{To}\right)\end{array}$ & $\begin{array}{c}\text { Dense } \\
\text { (Sito) }\left(\mathrm{So}_{\mathrm{T}} \mathrm{To}\right)\end{array}$ \\
\hline
\end{tabular}

\section{BIBLIOGRAPHIE}

CULIOLI Antoine, "Structuration d'une notion et typologie lexicale : à propos de la distinction dense, discret, compact” in : BULAG n 17 (pp. 7-12), Université de Franche-Comté, 1991-1992.

CULIOLI Antoine, Pour une linguistique de l'énonciation, 3 vol. Ophrys, Gap (1990-1999)

De VOGÜÉ Sarah, « Discret, dense, compact : les enjeux énonciatifs d'une typologie lexicale », in : La notion de prédicat, éd. JJ. Franckel, collection ERA 642, Université de Paris VII 1989.

FRANCKEL Jean-Jacques, « La préfixation en re - » in : Étude de quelques marqueurs aspectuels du francais, Droz 1989 (pp. 236-244)

FRANCKEL Jean-Jacques et PAILLARD Denis, « Discret, dense, compact : vers une typologie opératoire », in : Travaux de linguistique et de philologie XXIX, Stasbourg-Nancy 1991 (pp. 103-135).

JALENQUES Pierre, «Étude sémantique du préfixe RE en français contemporain » in : Langue Française $\mathrm{N}^{\circ} 133$, Hachette-Larousse 2002

JALENQUES Pierre, « Quand la diachronie renvoie à la synchronie : étude des emplois idiomatiques du préfixe RE en français (reconnaitre, remarquer, regarder, etc. ", in : Recherches linguistiques de Vincennes, $2001 \mathrm{n}^{\circ} 30$, (pp. 39-61).

KIBBEE Douglas A., “Assertion / atténuation ; subjectivité / objectivité en anglais et en français : seem / sembler", in : Relations discursives et traduction, Michel Ballard éd. (pp. 73-87), PUL Lille, 1995

SOLATIE Raija, «Verbes psychologiques dans une construction impersonnelle : analyse contrastive allemand-français ", in :Nouveaux Cahiers d'Allemand, (Association des -) 1996 n $^{\circ} 4$

THUILLIER François, Systématique des emplois du verbe "paraître" en français contemporain, Thèse de doctorat, Université de Paris VII, 2003 (300p) , $\mathrm{n}^{\circ}$ national de thèse : 2003PA070014

\section{NOTES}

1. On pourrait appeler ces termes $\mathrm{C} 0$ et $\mathrm{C} 1$, respectivement. Mais les termes "source, but » évoquent mieux la relation entre la réalité qui «donne» l'impression et l'observateur qui « reçoit » cette impression.

2. Notons cependant qu'en français québécois, on assiste à une neutralisation de cette distinction : 
Tu as l'air d'avoir reçu du courrier (FF) * Ça a l'air que tu as reçu du courrier

Il semble que tu aies reçu du courrier (FQ) Ça a l'air que tu as reçu du courrier

3. Dans le cas des expressions météorologiques, l'impersonnel renvoie à l'existence du cosmos dont l'énonciateur prend conscience avant de formuler une qualification à son sujet:

Il fait un temps beau et frais Le temps est beau et frais

Il fait beau et frais

Il fait du soleil, du vent Le temps est au soleil, au vent

Les expressions à sujet défini (à droite ci-dessus) marquent au contraire un sujet déjà ancré dans la situation d'énonciation, l'article défini renvoyant à une première mention.

4. Seul le contraste présent / imparfait est possible, mais l'imparfait n'est qu'un inaccompli transposé dans le passé. Il s'agit donc là, non d'un contraste aspectuel, mais d'un contraste temporel tel qu'on envisage ou non la coïncidence entre le moment-origine To et le moment de locution T1.

5. Je souligne que cette notation personnelle des paramètres de l'énonciation diffère de celle qui est couramment utilisée dans la littérature culiolienne, pour une quantité de raisons que je ne peux développer ici, faute de place. Disons que dans ma notation, So est le paramètre qui permet de travailler sur la classe des sujets (S, S'), To celui qui permet de travailler sur la classe des instants $\left(T, T^{\prime}\right)$. Ces deux paramètres définissent conjointement le point de vue QNT. Et Sito est le paramètre qui définit le point de vue QLT et permet de travailler sur la classe des états (P, $\left.\mathrm{P}^{\prime}\right)$. Mais il reste entendu que ces trois paramètres définissent solidairement ce qu'on appelle «le point de vue de l'énonciateur ».

6. Voir aussi les expressions où «faire » traduit une sensation d'origine météorologique, où on a le choix entre un point de vue subjectif (procès construit à partir de $\mathrm{S}$, l'expérienceur qui éprouve la sensation) et un point de vue objectif (l'expérienceur $\mathrm{S}$ s'efface et le procès est construit à partir d'un impersonnel représentant le cosmos) :

$<\mathrm{SVY}><() \mathrm{r} \mathrm{Y}\rangle$

je vois des éclairs il fait des éclairs

j'entends l'orage il fait de l'orage

je sens le vent il fait du vent

j'ai froid, chaud il fait froid, chaud

7. A. Culioli (1991) écrit ceci : «Le dense correspond à un mixte, un cas intermédiaire et instable [entre le discret et le compact ]. Ni QNT, ni QLT ne sont prépondérants ». Je rappelle que dans ma notation, QLT est Sito et QNT est le couple So, To. Par ailleurs, je signale que JJ. Franckel et D. Paillard (1991) ont une conception de la pondération qui diffère de celle d'A. Culioli.

8. Ce «lissage" notionnel rappelle la densité de l'ensemble des nombres rationnels en mathématiques: entre 1 et 2 , il existe une infinité de valeurs : de 1,1 à 1,9 , mais aussi de 1,11 à 1,19 et de 1,91 à 1,99 , etc.

9. Sauf « sembler » qui ne prend pas de préposition ou qui demande le connecteur « que ». Peutêtre pourrait-on attribuer ceci au fait que la relation est établie en co-énonciation.

10. Cette définition classique n'apparaît pas chez S. de Voguë (1989), mais elle correspond à la différence entre les schémas pp. 20 et 21 , où ce qui est neutralisé est bel et bien la différence entre $\mathrm{p}^{\circ}$ et $\overline{\mathrm{p}}^{\circ}$

11. Je ne peux développer, faute de place, mais ceci embrasse une quantité de phénomènes connus. Ainsi, avec un verbe dense, l'agentivité reste présente, quoi qu'on fasse : "Marie est fouettée " = on la fouette. Alors qu'avec un verbe discret, cette agentivité peut s'éclipser totalement au profit de l'état du but : « Marie est assommée » = elle est inconsciente. 


\section{RÉSUMÉS}

En français, l'idée d'apparence peut s'exprimer de diverses manières, notamment avec « sembler, avoir l'air, ressembler, faire ». J'ai essayé de rendre compte des différences de sens et d'usage de ces verbes dans la perspective de la Théorie des Opérations Prédicatives et Énonciatives. J'ai proposé de représenter chacun de ces verbes par un schéma de lexis spécifique, à partir duquel on peut calculer toutes les propriétés distributionnelles de chacun. Plus précisément, chaque schéma correspond à une pondération spécifique entre les paramètres de l'énonciation. C'est ainsi que le domaine notionnel de l'énoncé global peut être caractérisé, en termes topologiques, comme discret / dense / compact.

In French, the idea of appearance can be expressed in various ways, in particular with "sembler, avoir l'air, ressembler, faire". I have tried to account for the semantic and syntactic differences between these verbs in the perspective of the "Théorie des Opérations Prédicatives et Énonciatives". I have proposed to represent each of these verbs through a specific lexis pattern, from which all the distributional proprieties can be calculated. More precisely, each pattern corresponds to a specific ponderation between the parameters of enunciation. Thus, the notional domain of the whole sentence can be characterized in terms of discreet / dense / compact topology.

\section{INDEX}

Mots-clés : Schéma de lexis, paramètres de l'énonciation, pondération, topologie, discret, dense, compact

Keywords : lexis pattern, parameters of enunciation, ponderation, discreet / dense / compact topology.

\section{AUTEUR}

\section{ALAIN DELPLANQUE}

Université François-Rabelais (Tours), Équipe « Langues et représentations » 